\title{
Catalogue of Ap, HgMn and Am stars ${ }^{\star}$
}

\author{
P. Renson and J. Manfroid ${ }^{\star \star}$
}

\author{
Institut d'Astrophysique et de Géophysique, Université de Liège, Allée du 6 août 17, 4000 Liège, Belgium \\ e-mail: manfroid@astro.ulg.ac.be
}

Received 12 August 2008 / Accepted 17 February 2009

\section{ABSTRACT}

We present a catalogue of 8205 known or suspected Ap, HgMn and Am stars. This paper is a major update of our first edition of the catalog of Ap and Am stars and includes revised identifications, additional stars and revised information obtained from the literature.

Key words. catalogs - stars: chemically peculiar

\section{Introduction}

A large amount of observational data has been collected on Ap, HgMn and Am stars over the last decades. Still, there remain many unsolved issues concerning the phenomena they harbor. Pursuing the study of these stars seems thus necessary and a catalogue compiling their known characteristics is a valuable tool. Since the first edition of such a catalogue (Renson et al. 1991), new Ap, HgMn and Am stars have been recognized in various works, e.g., in the fifth Michigan catalogue (Houk \& Swift 1999). Others have been discovered in observational surveys, mainly in relatively distant open clusters (see, e.g., compilations by Bayer et al. 2000; Paunzen \& Maitzen 2001, 2002; Paunzen et al. 2002, 2003, 2005, 2006; Netopil et al. 2007). and in other searches, such as a photometric study based on the Strömgren-Crawford indices (Masana et al. 1998) yielding 60 new CP candidates, the more than $500 \mathrm{Ap}$ and Am stars discovered at Abastumani Astrophysical Observatory in the course of classifying stars in the galactic anticenter direction (Kharadze \& Chargeishvili 1990) or the study by Radoslavova (1989) in the direction of the OB associations Cygnus OB4, Cepheus-Lacerta OB1 and Cassiopeia OB9.

New data have been gathered, such as new periods of Ap stars (see, e.g, compilations by Catalano et al. 1991, 1993; Catalano \& Renson 1997, 1998; Renson \& Catalano 2001). Very strong magnetic fields have been discovered in some Ap stars (see, e.g., Babel et al. 1995; Babel \& North 1997; Bagnulo et al. 2003; Hubrig et al. 2005) and considerable efforts have been brought into the analysis of magnetic fields (e.g., Mathys 1991, 1995; Mathys \& Lanz 1992; Mathys \& Hubrig 1997; Mathys et al. 1997; Hubrig et al. 2000, 2004, 2005, 2006; Aurière et al. 2007; Wade et al. 2000; and the catalogue by Bychkov et al. 2003, and references therein).

\section{Description}

The catalogue includes 8205 stars (vs. 6684 for the first edition), among which 3652 Ap stars (or probably Ap), $162 \mathrm{HgMn}$ (or

\footnotetext{
* Catalogue (full Table 1) is only available in electronic form at the CDS via anonymous ftp to

cdsarc.u-strasbg.fr (130.79.128.5) or via

http://cdsweb.u-strasbg.fr/cgi-bin/qcat?J/A+A/498/961

$\star \star$ Research Director FNRS.
}

probable) and 4299 Am (or probable), and 92 stars which have been wrongly catalogued at least once as Ap, HgMn or Am.

As for the first edition, all stars are grouped together in a single list. The new name of the catalogue reflects the fact that, according to current usage, $\mathrm{HgMn}$ are no longer referred to as Ap stars. The category "Ap star" is taken in the broadest sense. It also includes Bp stars, in particular the He-weak and He-strong stars. Similarly, we include the Fm stars under the Am banner. Those having a $\delta$ Del type are mentioned. However, the chemically peculiar stars having abundance anomalies resulting from selective accretion of circumstellar material, i.e., the weak-lined $\lambda$ Boo stars, are not included.

Whenever possible, the data included in the catalogue have been taken from original papers in order to avoid faulty ratifications of existing misprints and other errors, some being repeated from paper to paper. This is especially true for components of double stars as it is often difficult to establish whether the data found in a paper or a table relate to the whole system or to one component and to which one.

The data have been collected according to the same principles as for the first edition (Renson et al. 1991). The columns of the table are mostly the same. Table 1 shows the first records of the catalogue.

The following identificators are given: the HD number (Cannon \& Pickering 1918-24) or HDE number (Cannon 1925-36; Cannon \& Mayall 1949), the HR number (Pickering et al. 1908), most generally used for bright stars since it serves as a running number in all editions of the Bright Star Catalogue, a Durchmusterung number, the designation (Greek or Latin letter, or number) of the star in its constellation.

Hipparcos and Tycho numbers have been added wherever possible. Bertaud's number (Bertaud 1959, 1960, 1965; and Bertaud \& Floquet 1974) and GC numbers (Boss 1937) have been dropped. However, the latter appear in the $\mathrm{N}$ notes when no HIP designation is available, or, for multiple stars, in the D notes when the GC numbers of the components are different.

Additional identifications for some stars can also be found in the $\mathrm{N}$ notes, e.g., another Durchmusterung number, the [B10] numbers from the preliminary general catalogue by Lewis Boss (1910) and the GCRV numbers from the catalogue of radial velocities by Wilson (1953). These two catalogues (previously designated as $\mathrm{B}$ and $\mathrm{W}$ ) have been used as the only identifiers in older works. 
Table 1. First records of the catalogue. The full table is available at the CDS.

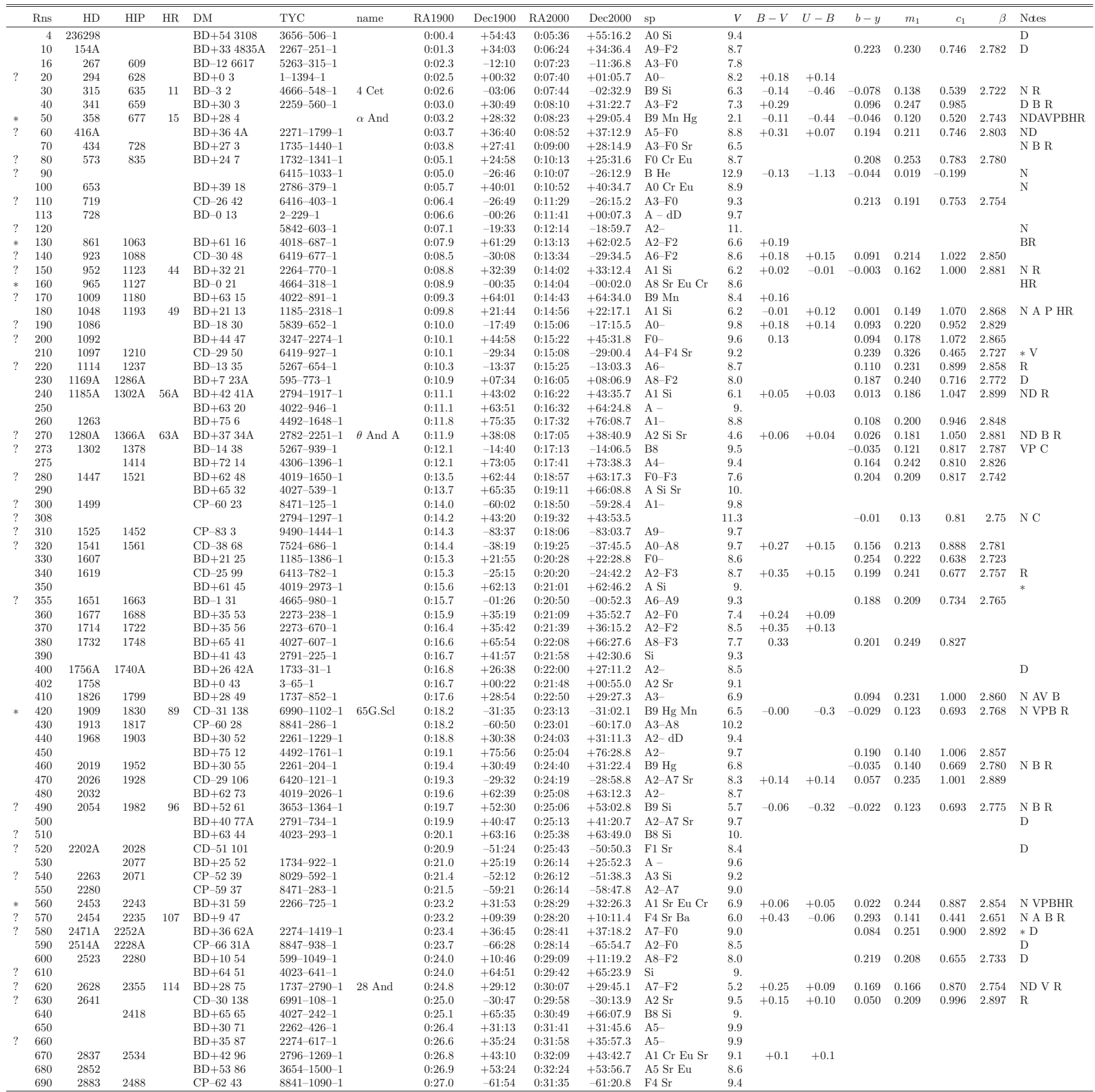

The first column indicates the degree of confidence in the peculiarity character. The next columns are identifiers (HD, HIP, HR, DM, TYC, and the designation in the constellation), the 1900 and 2000 coordinates, the spectrum and Johnson and Strömgren photometric data. The last column refers to additional notes. See text for more information.

The 1950 coordinates listed in the previous edition are dropped in favour of the 2000 coordinates. They are obtained from the Hipparcos, Tycho or 2MASS catalogues, after carefully checking the identification, and are listed with a better precision than in the first edition, thus removing any ambiguity.

The running numbers, often quoted as Rns in the literature, have been kept for the stars already present in the first edition. Initially these numbers were chosen as successive multiples of 10, allowing the insertion of additional stars. This preliminary list was presented at the 19th meeting of the European Workgroup on Ap Stars and the assigned numbers have then been considered as definitive. Because of delays in its publication, the first edition already included 500 new insertions.

The Rns numbers closely follow the HD order or, in the absence of HD number, the 1900 right ascension.

The stars added for the present edition have been inserted between the existing ones, following the same scheme. Obviously there were several instances where 9 slots were not sufficient (e.g., clusters, Kharadze's field), and the 1900 RA order could not be always exactly obeyed. The extra precision on the right ascension revealed that some stars present in the first edition were not ordered perfectly. There are necessarily a few more 
such instances in the present edition. On the other hand it has still been possible to strictly respect the HD order.

Details about multiplicity are found in the D notes. For a double or multiple star the ADS number (Aitken 1932) if any, or sometimes the BDS number (Burnham 1906), is given in note D, in which the distance(s) and magnitude(s) of the other component(s) are given. The identifiers (HD, HR, DM, HIP) of multiple stars are followed by a letter $(\mathrm{A}, \mathrm{B}, \ldots)$ unless the magnitude of the other components is too faint $(\Delta m \geq 5)$ or the angular separation is too large. The absence of such a letter does not mean the absence of a faint or still undiscovered companion. This is especially true for the fainter stars of the catalogue. Similarly, spectroscopic binaries may still be undetected (absence of B note). This can be the case for stars included on the basis of purely photometric criteria (notes C: Str., Gen., Mtz., ...) for which no indication of radial velocity variations exist.

The next data are the magnitude and Johnson's colour indices $B-V$ and $U-B$. For most stars the given magnitude is $V$ in Johnson's system or $y$ in Strömgren's one, but for less luminous stars it can be Tycho $V$, or an approximate value and sometimes a photographic magnitude. For close doubles (except for very close ones detected only by speckle interferometry, etc.) the magnitude is corrected for the light of the companion. This is never done for the colour indices. No correction is introduced for interstellar absorption and reddening.

The numerical data given for each star such as magnitude and colour, spectral type, projected rotational velocity, etc. are weighted means of values found in the literature. For the spectral types, the HD catalogue (and the Michigan Spectral Catalogue for southern stars) is always consulted as well as specific papers. All the numerical values quoted are given for information only, without claiming a high precision.

A flag indicates the degree of confidence for the $\mathrm{CP}$ nature of the stars. A slash (/) denotes a star that was improperly considered to have an Ap, $\mathrm{HgMn}$ or Am nature. Question marks (?) mean doubtful cases (2314 stars, among which are 311 Ap stars detected by photometric criteria, reported in notes $\mathrm{C}$, and not yet confirmed by ordinary spectroscopy). On the contrary asterisks (*) denote well-known confirmed Ap, HgMn or Am stars (only 426).

Notes A indicate the cluster, group or association to which the stars belong. Another identificator of when the star is known to vary is the variable-star name. It is then given in note V. The period of variation, which the rotator model considers to be the rotation period, is quoted in note $\mathrm{P}$, with the indication of the quantities observed to vary with that period (luminosity, spectral line intensities, magnetic field, ...). The period is to be read in note $\mathrm{B}$ if the variation originates from an orbital motion (eclipses, ...). The presence of note B means that eclipses or radial-velocity variations have been observed and, whenever possible, the period (and sometimes the eccentricity) is given. If the star received a variable-star name because of oscillations or pulsations such as in $\delta$ Sct variables, it is indicated in note $\mathrm{V}$ itself. Other notes give the effective magnetic field (L), the mean modulus of the field $(\mathrm{M})$ and the quadratic field $(\mathrm{Q})$.

For Ap stars the most conspicuously overabundant elements are indicated by their chemical symbols besides the spectral type. The K-line type and the metallic-line type are both given for Am stars, separated by a hyphen. If only one type is known, the hyphen nevertheless appears, so that it is always the test to distinguish Ap and Am stars. The $\delta$ Del type stars are included among the Am stars, but are marked with dD. Comments about the spectral type may sometimes be found in notes *, e.g., the

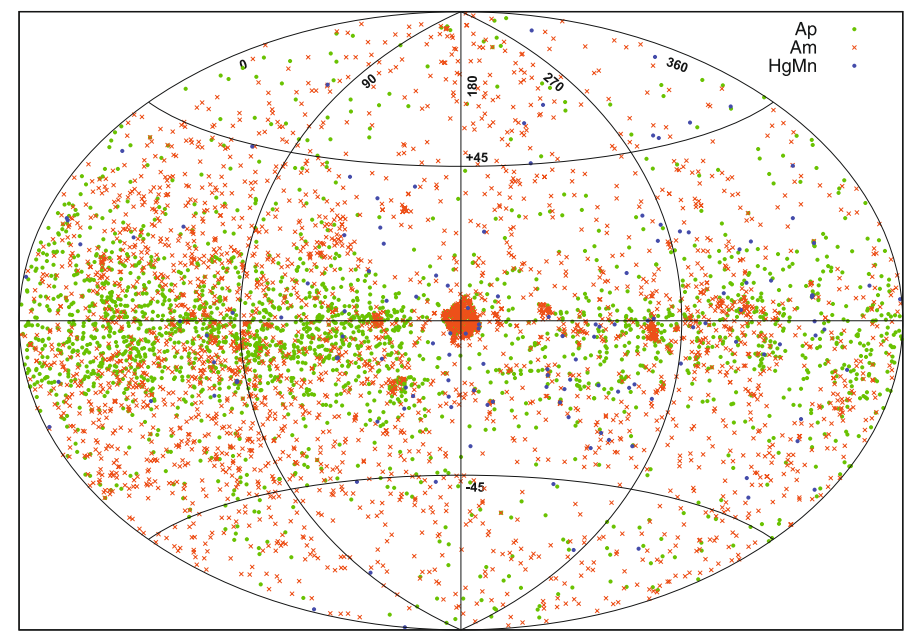

Fig. 1. Distribution of Ap, $\mathrm{HgMn}$ and Am stars in galactic coordinates. The chart is centered on the galactic anticenter, $l=180$, where the density of identified CP stars is highest thanks to Kharadze \& Chargeishvili's survey (1990).

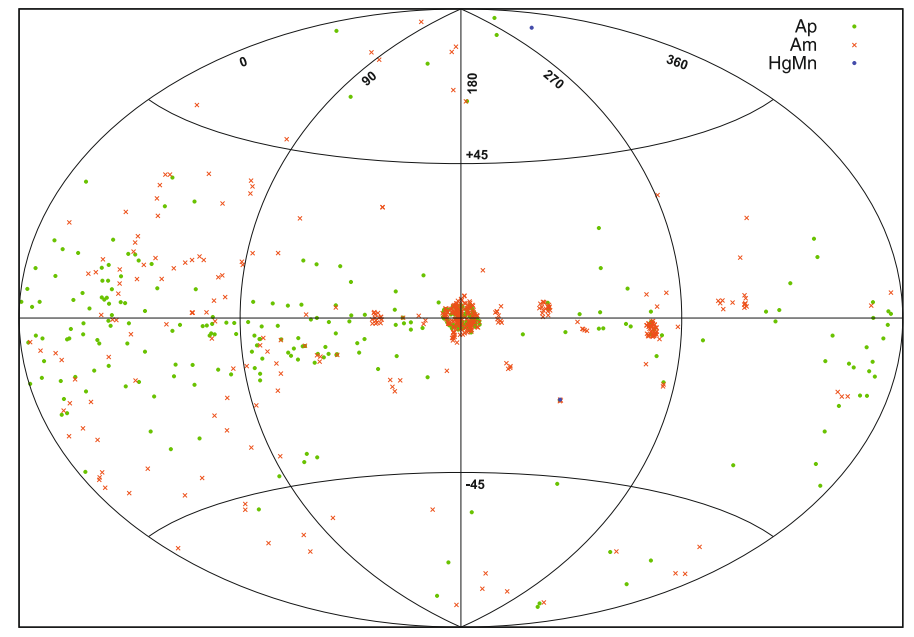

Fig. 2. Distribution of faint $(V>10) \mathrm{Ap}, \mathrm{HgMn}$ and Am stars in galactic coordinates.

fact that an author found an Ap type for an Am star or vice versa. However these notes may include other remarks.

\section{Analysis}

The catalogue is far from homogeneous and there remains considerable observational biases relative to several parameters. As such it is not well suited to perform statistical analyses. We nevertheless illustrate its content with several informative plots.

The spatial distribution of stars is shown in Figs. 1, 2. The darkest patches mainly correspond to areas where the greatest observational efforts have been done, rather than actual concentrations of Ap and Am stars. This is particularly true around the galactic anticenter. The galactic equator is prominent mainly for the Ap stars - which are more luminous and globally more distant - and several galactic clusters can be identified.

The color-magnitude diagram (Fig. 3) is given for stars having parallaxes with relative errors smaller than 0.1 . Different symbols are used for stars with different spectral characteristics (Si, HgMn, EuSrCr, Am, etc.). These results concern relatively nearby stars with accurate data and, consequently, small samples. Larger samples can be used for color-color $(U-B, B-V)$ 


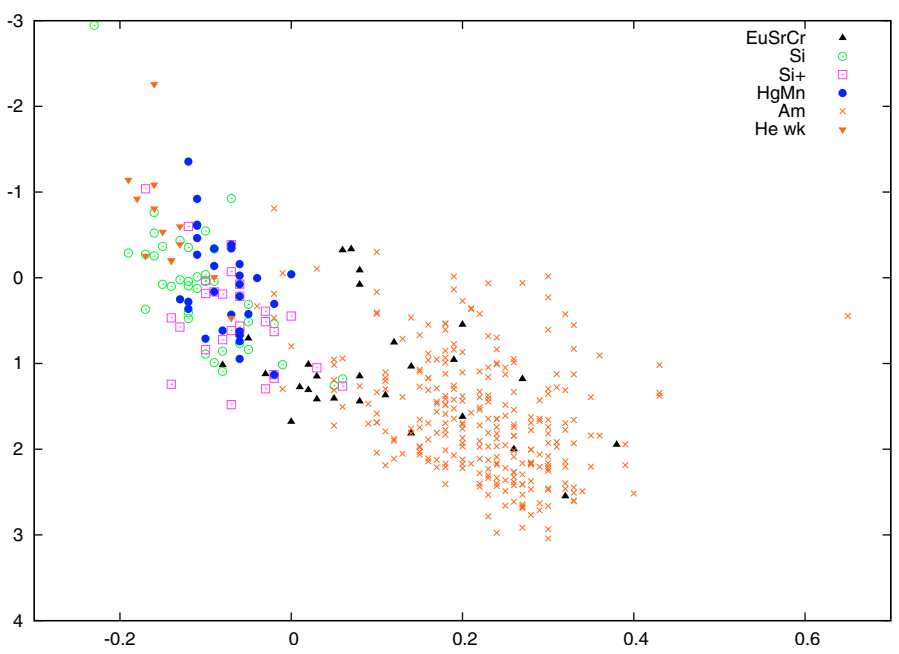

Fig. 3. Absolute $V$ magnitude as a function of $B-V$ for different groups of stars. ApSi with and without additional elements have distinct symbols.

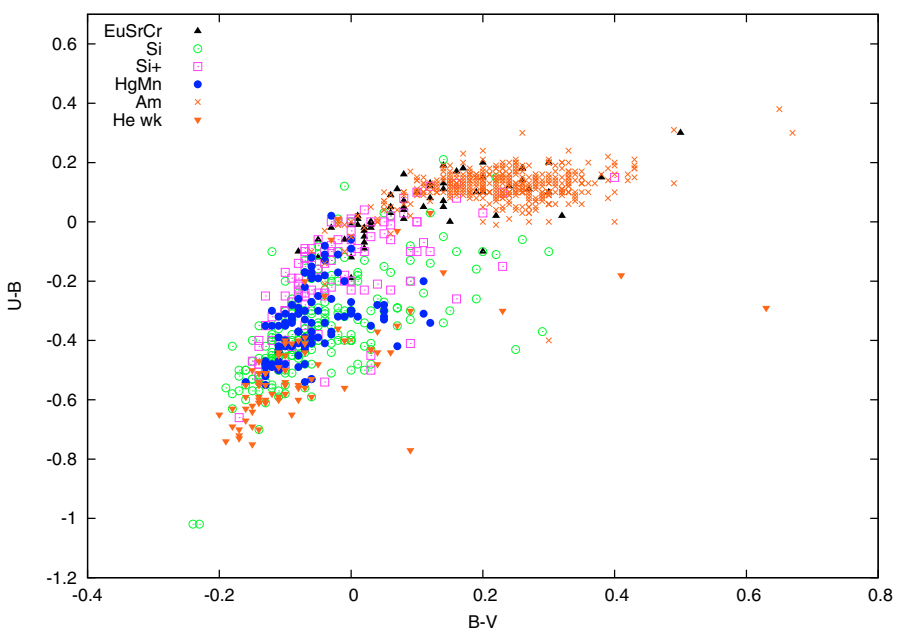

Fig. 4. Colour-colour diagram for stars brighter than $V=9$.

diagrams (Fig. 4). The scatter is larger since the accuracy for the fainter stars is worse, and extinction effects are not accounted for.

The local space density of confirmed and candidate (or uncertain) Ap and Am stars is shown in Figs. 5 and 6. Only stars with a relative parallax error smaller than 0.2 are included. The density of well-established Am stars declines rather sharply above $\log (d) \sim 1.8$, i.e., 60 pc. The density of Ap stars shows a plateau up to $\log (d) \sim 2.15$, i.e., $140 \mathrm{pc}$. Counts for the less numerous $\mathrm{HgMn}$ are not meaningful below $\log (d) \sim 1.8$. Above this they show a plateau similar to the Ap. Perhaps not coincidentally, those distances correspond to an apparent magnitude $V \sim 6$, which shows that the catalogue is at best complete only for the very brightest stars. These spheres include only $103 \mathrm{Am}$, $45 \mathrm{HgMn}$ and $178 \mathrm{Ap}$ stars. The respective average densities are $1.0 \times 10^{-4} \mathrm{pc}^{-3}, 4.0 \times 10^{-6} \mathrm{pc}^{-3}$ and $1.5 \times 10^{-5} \mathrm{pc}^{-3}$. Confirmation of a fraction of the candidates would significantly boost those values.

The rotational periods are the subject of Figs. 7, 8. The histogram shows the well-known peaked distribution with a maximum around 2 days. No correlation of the period with the color index can be found.

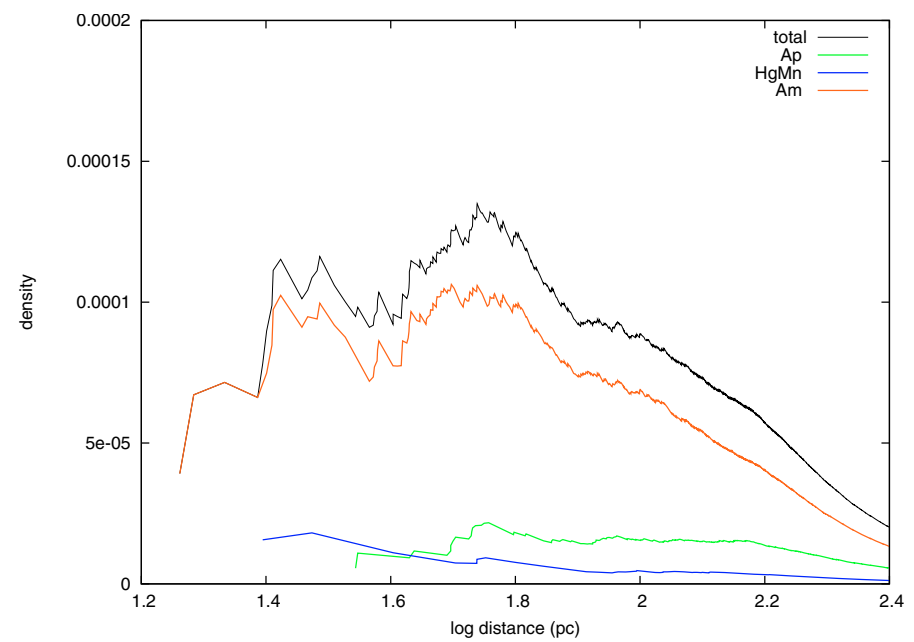

Fig. 5. Average space density in $\mathrm{pc}^{-3}$ of confirmed Ap, $\mathrm{HgMn}$ and Am stars within a sphere of a given radius, up to about $250 \mathrm{pc}$.

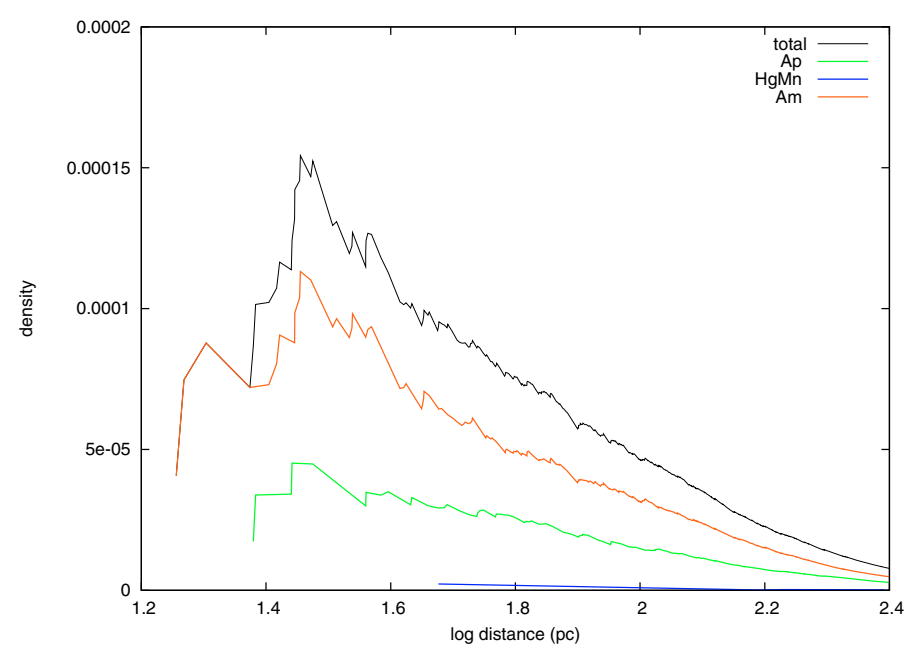

Fig. 6. Average space density of candidate Ap, $\mathrm{HgMn}$ and Am stars.

The number of known variables among helium-weak stars has significantly increased from a single one (Landis et al. 1985) or 15 (Renson 1985) to 39 out of a total of 111 stars.

Eclipsing Ap-stars are still uncommon. Their spectral variations during the eclipses could help map the stellar surface (see, e.g., Renson \& Mathys 1984). However, their primary interest is the possibility to determine unambiguously the fundamental parameters of the components, which in turn allow one to derive other constraints, such as their evolutionary status. Table 2 lists the eclipsing Ap-stars of the catalogue, including those with a "?", i.e., of a still doubtful Ap nature. We excluded the well-known Ap star 3 Cen A=V983 Cen (Rns 34750), because the presence of eclipses is not well established. Among the 15 remaining stars, 8 are flagged with a "?". Moreover, V883 Cen=HR 5292 (Rns 35356) is a poor candidate for the suggested analysis since the Ap star is the secondary component and its spectrum is diluted by the much brighter B5 primary. IU Lup (Rns 37610) is also doubtful because it is perhaps a $\delta$ Del type star rather than an Fp Sr. Thus, only 5 bona fide candidates remain: AR Aur (Rns 8740), V414 Pup (18190), AO Vel (19090), HY Vir (33030), V892 Cen (34910).

Not surprisingly, the eclipsing Am are much more frequent (73 in the catalogue). Similarly, the distribution of orbital periods (Fig. 9) among binaries confirms a lower proportion of 


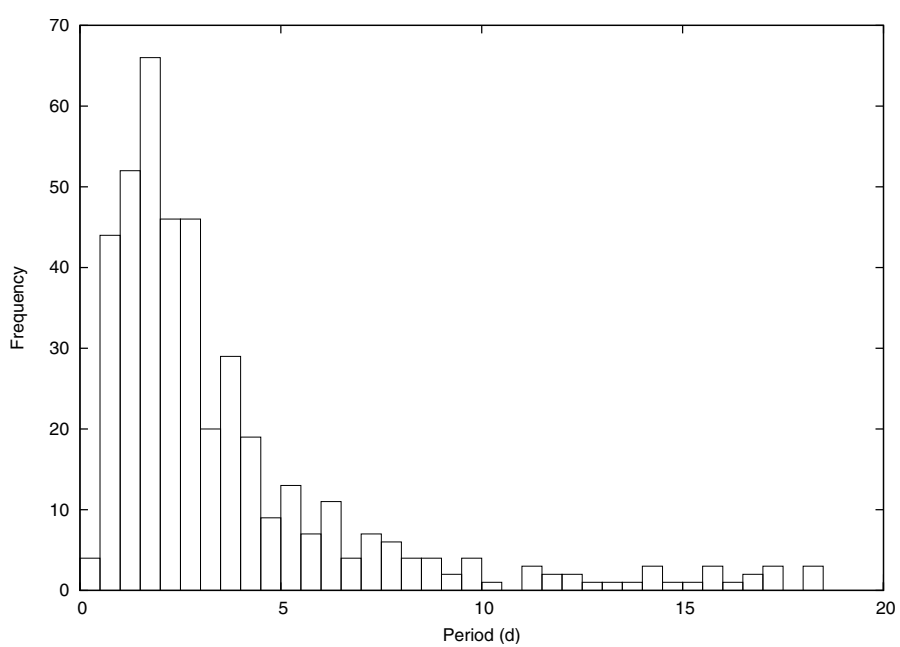

Fig. 7. Distribution of rotational periods of Ap stars below 20 days. Confirmed as well as candidate stars are included. When several periods are possible, only the first listed in the catalogue is considered.

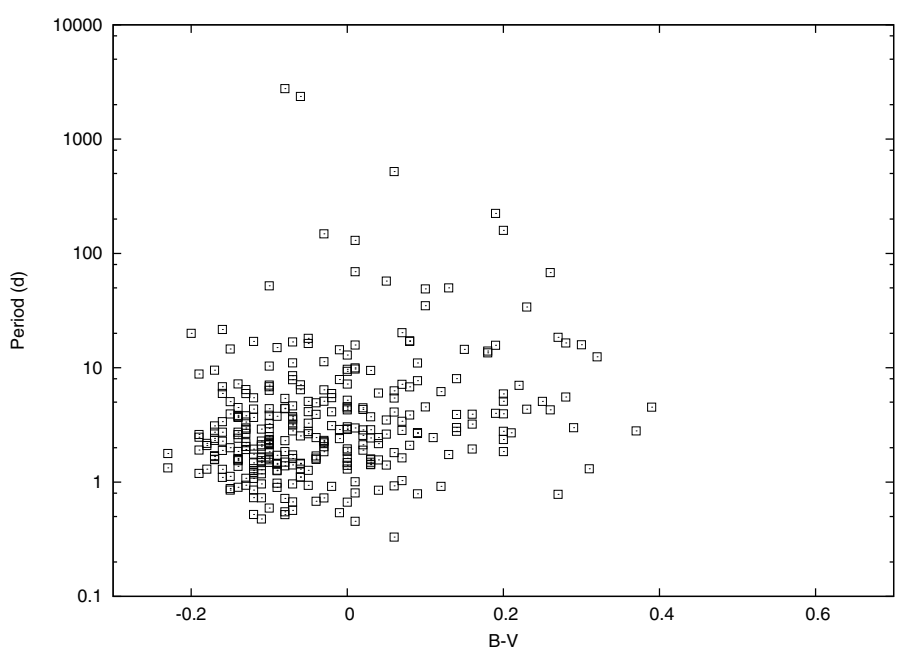

Fig. 8. Rotational periods of Ap stars as a function of $B-V$.

Table 2. Eclipsing variables among Ap. A question mark denotes star for which the Ap character is uncertain.

\begin{tabular}{lrlrlll}
\hline \hline Rns & HD & Name & $P(\mathrm{~d})$ & $V$ & Spectrum & Note \\
\hline 8740 & 34364 & AR Aur & 4.1347 & 6.1 & B9 Hg Mn & \\
12830 & 47755 & V684 Mon & 1.8514 & 8.4 & B6 & $?$ \\
18190 & 66051 & V414 Pup & 4.75 & 8.7 & A0 Si & \\
19090 & 68826 & AO Vel & 1.5847 & 9.3 & B9 Si & \\
26180 & 91021 & V349 Vel & $P=3.02$ & 9.7 & F3 Sr & $?$ \\
33030 & 114125 & HY Vir & 2.73234 & 7.8 & F2 Sr Eu Cr & \\
33790 & & AX Vir & 0.70253 & 10.3 & A2 & $?$ \\
34656 & 120166 & BF Vir & 0.6406 & 10.2 & A0 & $?$ \\
34910 & 121276 & V892 Cen & 6.5146 & 9.9 & A0 Si Cr He & \\
35356 & 123335 & V883 Cen & 35.45 & 7. & B He wk. Sr & \\
37610 & 132515 & IU Lup & 1.619 & 9.3 & F8 Sr & $?$ \\
37660 & 132742 & $\delta$ Lib & 2.32735 & 4.9 & A0 & $?$ \\
41470 & 146772 & CC Her & 1.734 & 10.2 & A1 Sr & $?$ \\
49600 & 178001 & BH Dra & 1.817 & 8.4 & A2+Ap & $?$ \\
50300 & 181615 & $v$ Sgr & 137.95 & 4.6 & B8 He & $?$ \\
\hline
\end{tabular}

tight orbits among the Ap class (see, e.g., North et al. 1998), and probably also the HgMn. Nevertheless, unlike previous surveys (see, e.g., North \& Debernardi 2004), the catalogue contains 5 sure cases of Ap stars with orbital periods shorter than 3 days.

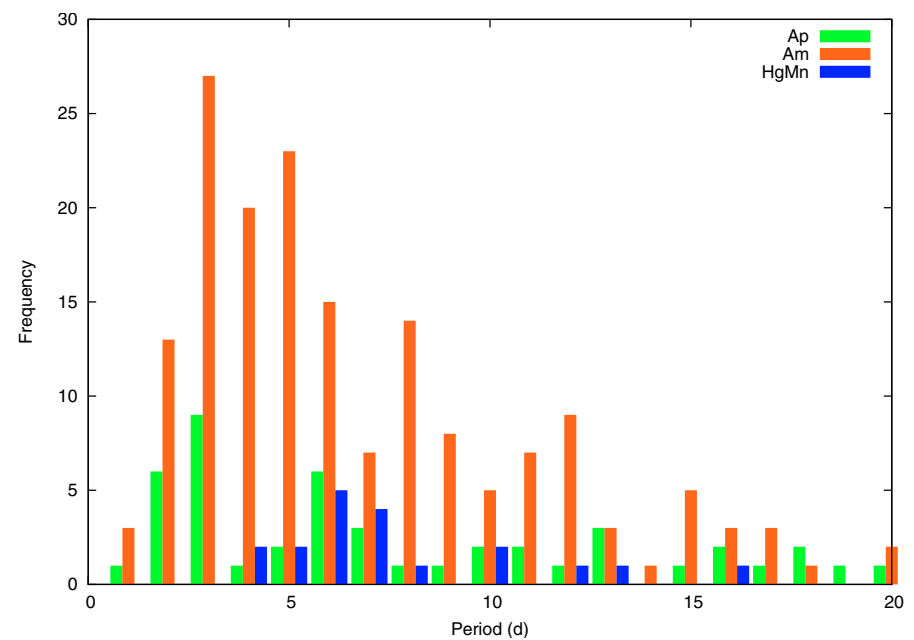

Fig. 9. Distribution of orbital periods below 20 days among the Ap, $\mathrm{HgMn}$ and Am binaries. Confirmed as well as candidate stars are included. The first bin corresponds to the interval $0.5-1.5 \mathrm{~d}$.

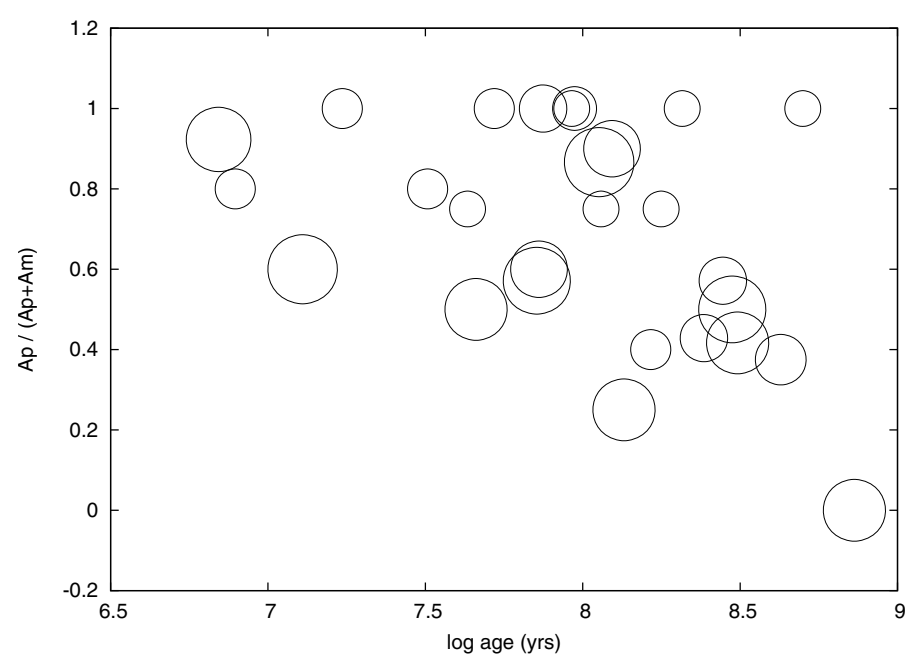

Fig. 10. Fraction of Ap stars among the CP stars in clusters, as a function of the age. The area of the circles is proportional to the number of stars included in the sample. The smallest circles contain 4 stars.

Additionally, there are 7 stars of uncertain Ap nature, and 3 of uncertain period.

Finally a rough correlation between the fraction of Ap stars among all CP stars in clusters and the age of the clusters can be deduced from Fig. 10, as already noticed by Renson (1971).

Acknowledgements. This research has made use of NASA's Astrophysics Data System Bibliographic Services, of the SIMBAD database, operated at CDS, Strasbourg, France, and of the WEBDA database, operated at the Institute for Astronomy of the University of Vienna. We would like to thank the referee Dr. G. Mathys for his valuable comments.

\section{References}

Aitken, R. G. 1932, New General Catalogue of Double Stars, Carnegie Inst. Washington, No. 417

Aurière, M., et al. 2007, A\&A, 475, 1053

Babel, J., \& North, P. 1997, A\&A, 325, 195

Babel, J., North, P., \& Queloz, D. 1995, A\&A, 303, L5

Bagnulo, S., Landstreet, J. D., Lo Curto, G., Szeifert, T., \& Wade, G. A. 2003, A\&A, 403, 645

Bayer, C., Maitzen, H. M., Paunzen, E., Rode-Paunzen, M., \& Sperl, M. 2000, A\&AS, 147, 99 
Bertaud, Ch. 1959, J. Obs., 42, 45

Bertaud, Ch. 1960, J. Obs., 43, 129

Bertaud, Ch. 1965, J. Obs., 48, 211

Bertaud, Ch., \& Floquet, M. 1974, A\&AS, 16, 71

Boss, B. 1937, General Catalogue of 33342 Stars for the Epoch 1950, Carnegie Institution of Washington Publication, 468, Washington, D.C.

Boss, L. 1910, Preliminary General Catalogue of 6188 Stars for the Epoch 1900, Carnegie Institution of Washington Publication, 115, Washington, D.C.

Burnham, S. W. 1906, General Catalogue of Double Stars within 121 degrees of the North Pole, Carnegie institution of Washington (Chicago: University of Chicago press)

Cannon, A. J. 1925-36, Ann. Astr. Ohs. Har. Coll., 100

Cannon, A. J., \& Mayall M. W. 1949, Ann. Astr. Obs. Har. Coll., 112

Cannon, A. J., \& Pickering, E. C. 1918-24, Ann. Astr. Obs. Har. Coll., 91

Bychkov, V. D., Bychkova, L. V., \& Madej, J. 2003, A\&A, 407, 631

Catalano, F. A., \& Renson, P. 1997, A\&AS, 121, 57

Catalano, F. A., \& Renson, P. 1998, A\&AS, 127, 421

Catalano, F. A., Renson, P., \& Leone, F. 1991, A\&AS, 87, 59

Catalano, F. A., Renson, P., \& Leone, F. 1993, A\&AS, 98, 269

Houk, N., \& Swift, C. 1999, Michigan catalogue of two-dimensional spectral types for the HD Stars, Ann Arbor, Michigan, 5

Hubrig, S., North, P., \& Mathys, G. 2000, ApJ, 539, 352

Hubrig, S., Szeifert, T., Schöller, M., Mathys, G., \& Kurtz, D. W. 2004, A\&A, 415,685

Hubrig, S., Nesvacil, N., Schöller, M., et al. 2005, A\&A, 440, L37

Hubrig, S., North, P., Schöller, M., \& Mathys, G. 2006, Astron. Nachr., 327, 289

Kharadze, E. K., \& Chargeishvili, K. B. 1990, AJ, 99, 379

Landis, H. J., Louth, H., \& Hall, D. S. 1985, IBVS, 2662, 1

Masana, E., Jordi, C., Maitzen, H. M., \& Torra, J. 1998, A\&AS, 128, 265
Mathys, G. 1991, A\&AS, 89, 121

Mathys, G. 1995, A\&A, 293, 746

Mathys, G., \& Hubrig, S. 1997, A\&AS, 124, 475

Mathys, G., \& Lanz, T. 1992, A\&A, 256, 169

Mathys, G., Hubrig, S., Landstreet, J. D., Lanz, T., \& Manfroid, J. 1997, A\&AS, 123,353

Netopil, M., Paunzen, E., Maitzen, et al. 2007, A\&A, 462, 591

North, P., \& Debernardi, Y. 2004, Spectroscopically and Spatially Resolving the Components of the Close Binary Stars, 318, 297

North, P., Ginestet, N., Carquillat, J.-M., Carrier, F., \& Udry, S. 1998, Contributions of the Astronomical Observatory Skalnate Pleso, 27, 179

Paunzen, E., \& Maitzen, H. M. 2001, A\&A, 373, 153

Paunzen, E., \& Maitzen, H. M. 2002, A\&A, 385, 867

Paunzen, E., Pintado, O. I., \& Maitzen, H. M. 2002, A\&A, 395, 823

Paunzen, E., Pintado, O. I., \& Maitzen, H. M. 2003, A\&A, 412, 721

Paunzen, E., Netopil, M., Iliev, I. K., et al. 2005, A\&A, 443, 157

Paunzen, E., Netopil, M., Iliev, I. K., et al. 2006, A\&A, 454, 171

Pickering, E. C. 1908, Revised Harvard Photometry, Ann. Astr. Obs. Har. Coll., 50

Radoslavova, Ts. 1989, Astrofiz.Issled., 5, 51

Renson, P. 1971, A\&A, 13, 130

Renson, P. 1985, IBVS, 2708,

Renson, P., \& Catalano, F. A. 2001, A\&A, 378, 113

Renson, P., \& Mathys, G. 1984, IBVS, 2522, 1

Renson, P., Gerbaldi, M., \& Catalano, F. A. 1991, A\&AS, 89, 429

Wade, G. A., Kudryavtsev, D., Romanyuk, I. I., Landstreet, J. D., \& Mathys, G. 2000, A\&A, 355, 1080

Wilson, R. E. 1953, General catalogue of stellar radial velocities, Carnegie Institution of Washington Publication, 601, Washington, D.C. 